\title{
Cyclopia - A Rare Lethal Malformation
}

\author{
Sunil Kumar Agarwalla', Upasana Patra' \\ ${ }^{1}$ Professor Paediatrics, Sishu Bhawan, Cuttack, Odisha \\ ${ }^{2}$ Junior Resident, Dept. of Paediatrics, MKCG Medical College, Berhampur, Odisha
}

Corresponding Author: Upasana Patra

\begin{abstract}
Cyclopia is the rare manifestation of most severe degree of alobar holoprosencephaly ${ }^{1}$. Its most cardinal feature is presence of single eye or partially divided eye in a single socket at mid face with varying degrees of fusion of globes. The teratogenic factors leading to this anomaly include genetics, drugs, infection, radiation. Very few reports have documented this anomaly in newborn. Hence we present a case of stillborn baby of cyclopia with synophthalmia to a 27 year old woman who presented in second stage of labour with USG showing placenta previa. Early prenatal diagnosis, genetic study and proper management of this anomaly should be emphasized for better outcome in future.
\end{abstract}

Keywords: cyclopia; holoprosencephaly; teratogenic; synophthalmia; prenatal diagnosis

\section{INTRODUCTION}

Cyclopia also known as cyclocephaly, is characterised by incomplete cleavage of prosencephalon into two separate hemispheres occurring between day 18 and day 28 of gestation. Its incidence is 1 in 1,00,000 newborns out of which $50 \%$ are stillborn and rest survive only for few hours ${ }^{2,3}$. There is preponderance of female babies born with cyclopia $^{4}$. It is a life threatening severe faciocerebral development deformity. So herewith, we present a rare case of cyclopic baby of a high risk mother having placenta previa to focus light on awareness regarding early prenatal diagnosis.

\section{CASE STUDY}

A still born female baby with dysmorphic facies having birth weight $2 \mathrm{~kg}$ was born of non consanguineous marriage at MKCG HOSPITAL, Berhampur on $16 / 6 / 2021$. It was a product of second pregnancy of a 27 year old woman G2P1L1A0, who was a registered case, did antenatal check up 8 times during her pregnancy, took 2 doses of $\mathrm{Td}$ with continuation of IFA and calcium supplementation. Her previous pregnancy was uneventful. She had no history of diabetes, hypertension, radiation exposure, fever, jaundice, UTI. Antenatal USG done at 25 weeks revealed low lying placenta previa of grade 1 maturation and dilated lateral ventricles cavum pellucidum was not fully visualised. On 16/06/2021, baby was born at 34 weeks gestation by caesarean section as there was no fetal movement perceived and mother was at high risk with low lying placenta previa. On gross examination, baby was stillborn and noted to have facial dysmorphism with peripheral cyanosis. There was presence of two fused eyeballs in a single orbit in the middle of the forehead, absence of nasal aperture, a projection like tubular appendage in centre of forehead suggestive of proboscis above eye(fig1), low set ear, umbilical cord and external genitalia were normal(fig2). No other extracranial congenital malformation was noticed. These typical apparent features are suggestive of cyclopia. Confirmation by chromosomal analysis and MRI could not be done as family did not give the consent. 


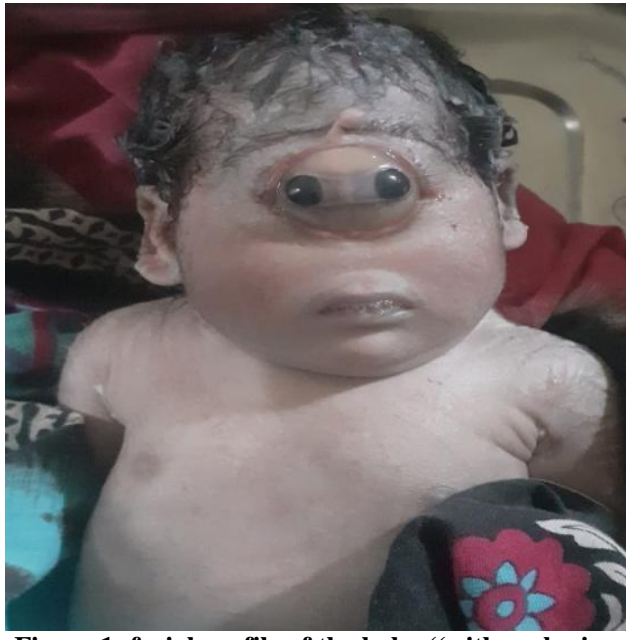

Figure 1- facial profile of the baby ((with cyclopia

\section{DISCUSSION}

The word "Cyclops" comes from the Greek "kyklos"(circle) + "ops"(eye) $)^{5}$. The term "cyclopia" is derived from mythical race of lawless giant shepherds in Greek mythology who had large round eye at the centre of forehead ${ }^{6}$. Cyclopia and other milder forms of developmental disorder result from holoprosencephaly which is failure of embryonic forebrain to divide into hemispheres properly.

Holoprosencephaly are of four types $\rightarrow$ Alobar (cyclopia), semilobar, lobar, middle interhemispheric variant holoprosencephaly, out of which cyclopia is the most severe form developing between day 18 and day 28 of pregnancy ${ }^{3}$. It occurs when some proteins are expressed inappropriately causing incomplete cleavage of brain into two distinct hemispheres. This results in fetus having one olfactory lobe with one eye and nasal malformations. SHH (Sonic hedgehod gene regulator) is the gene responsible for separation of single eye into two separate orbits. But when SHH gets mutated, there is fusion of eye in centre of face $^{7}$. Exact causes of cyclopia are unknown but possible heterogenous risk factors identified are genetic, chromosomal (mostly trisomy 13$)^{8}$, gestational diabetes, infections like TORCH, exposure to UV light, alcohol consumption and drugs like anticonvulsants, lithium, anticancer drugs, contraceptives during pregnancy or syndromic association

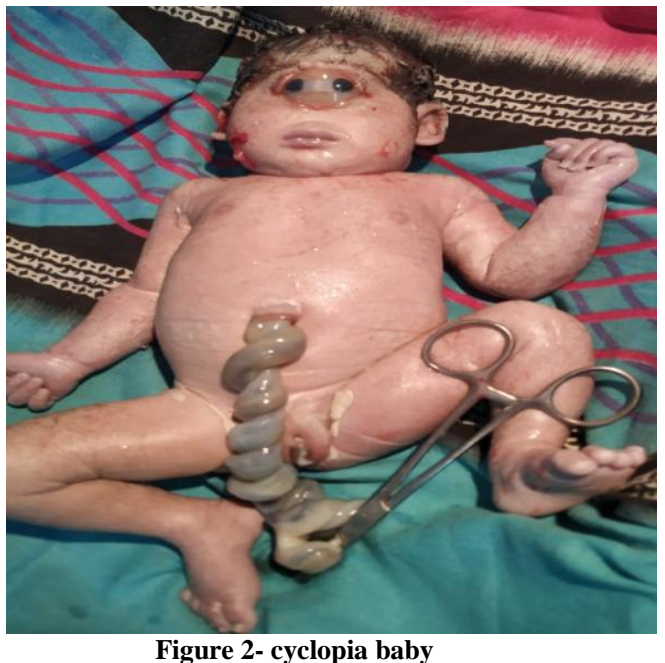

like Smith-Lemli-Opitz syndrome. Our patient had no such history of intake of drugs during pregnancy.

There are three types of eye deformities in cyclopia $\rightarrow$ monophthalmia (one eye), synophthalmia (two fused eye ball in a single orbit at the middle of face), or anophthalmia (complete absence of eyeballs). In this case, fetus had synophthalmia. Other distinct features include absence of nose, presence of proboscis (non-functioning nose) above eye, microcephaly, micrognathia, cleft palate, cleft lip, tooth abnormalities, genital abnormality ${ }^{3}$. Babies can have severe heart defects. The presence of extrafacial abnormalities like polydactyly, omphalocele, renal dysplasia constitute poor prognosis in cyclopia and associated with still birth ${ }^{9}$.

In our case intrauterine facial features of fetus passed undiagnosed. At birth our case was found to have typical facial features of cyclopia with synophthalmia, proboscis above the eye. Mother with placenta previa can be considered as a high risk factor for development of cyclopia, but no such reports have been documented till now. The case we describe here is the first case of cyclopia in a mother having placenta previa, though it could in fact be a mere coincidence. Since it is incompatible with life, so associated with neonatal mortality 
within few hours of birth, miscarriages, and stillbirths, thus not encountered in clinical practice.

In developing countries like ours where women do not receive antenatal checkups and do not undergo prenatal diagnosis, such cases go undetected. There is no way to prevent the condition and currently no cure is available. Careful intrauterine scanning of face by USG or fetal MRI at 11-13 weeks of gestation will enable early diagnosis and termination of pregnancy within recommended safe limit of gestational age.

\section{CONCLUSION}

Prognosis of cyclopia is grave. Conversation with a genetic counsellor and a paediatrician about this matter is important. Always look for congenital malformation associated with this anomaly. Early prenatal diagnosis of cyclopia and awareness regarding features of its sonographic findings can improve accuracy and have better outcome in future. Timely diagnosis and termination of pregnancy within safe limit is crucial to prevent complications and psychological affection in mother.

\section{Acknowledgement: None \\ Conflict of Interest: None \\ Source of Funding: None}

\section{REFERENCES}

1. Orioli IM, Amar E, Bakker MK, et al. Cyclopia: an epidemiologic study in a large dataset from the international clearing house of birth defects Surveillance and Research. Am J Med Genet C Semin Med Genet 2011; 157C:344-57.

2. Kallen B, Castilla EE , Lancaster PA, et al. The Cyclops and the mermaid: an epidemiological study of two types of rare malformation. J Med Genet 1992; 29:30-5.

3. Cannistra C, Barbet P, Parisi P, Iannetti G. Cyclopia: radiological and anatomical craniofacial post mortem study. J Craniomaxilloface Surg.2001;29(3):152-5

4. Ghassan SA Salama, Mahmoud AF Kaabneh, Mohamed K Al-Raqad et al. Cyclopia: A Rare Condition with Unusual Presentation - A Case Report. Clin Med Insights Pediatr. 2015; 9: 19-23. doi: 10.4137/CMPed.S21107

5. Bakker,Egbert J, The Meaning of meat and the structure of the Odyssey, Cambridge University Press,2013.ISBN 978-0-52111120-1.

6. Bremmer, J.N (1987).Odysseus versus the Cyclops, in Myth and Symbol. The Norwegian Institute.

7. Roessler E, Muenke M. The Molecular genetics of holoprosencephaly. Am J Med Genet C 2010; 154 C :52-61.

8. Poenaru MO, Vilcea ID, Marin A. Holoprosencephaly : two case reports. Maedica (Buchar). 2012 Jan ; 7(1) : 58-62.

9. Mc Gahan JP, Nyberg DA, Mack LA. Sonography of facia features of alobar and semilobar holoprosencephaly. Am J Roentgenol.1990;154(1):143-8

How to cite this article: Agarwalla SK, Patra U. Cyclopia - a rare lethal malformation. Int $J$ Health Sci Res. 2021; 11(11): 112-114. DOI: https://doi.org/10.52403/ijhsr.20211113 